\title{
Acute pericarditis as presenting symptom of staphylococcal endocarditis: Mitral valve involvement with fistulous tract from LV to LA and subsequent pseudoaneurysm development ${ }^{*}$
}

\author{
Eric McWilliams, Smriti Saraf, Katarzyna Dickinson
}

Cardiology Unit, Conquest Hospital, Hastings, UK

Email: eric.mcwilliams@gmail.com

Received 9 November 2012; revised 15 December 2012; accepted 23 December 2012

\begin{abstract}
Acute pericarditis is an unusual presentation of bacterial endocarditis [1]. It is most commonly associated with staphylococcal aureus infection and more likely to occur in young males in association with the risk factors of alcohol or substance abuse or diabetes. Tamponade is a common presenting feature and the aortic valve is the most commonly involved valve. This condition carries a very high mortality whether treated with antibiotics alone or in combination with surgery.
\end{abstract}

Keywords: Pericarditis; Endocarditis; Computed Tomography

\section{INTRODUCTION}

We report on an 82-year-old lady admitted with a history of dull central pain of 48 hours duration who was febrile and tachycardic. Her past medical history included asthma, previous transient ischemic attack, non ST segment elevation infarction with subsequent stenting of the LAD and a left total knee replacement, 9 months earlier, which was complicated by recurrent superficial wound cellulitis, treated by repeated courses of prolonged oral antibiotics. Clinically, venous pressure was elevated and there was dullness in the left base. White cell count was 11.5 and $\mathrm{C}$ reactive protein elevated at $282 \mathrm{mg} / \mathrm{dL}$ (ref $<2 \mathrm{mg} / \mathrm{dL}$ ). Chest $\mathrm{X}$ ray showed cardiomegaly with a small left pleural effusion (Figure 1(a)) EKG revealed sinus tachycardia with diffuse ST segment elevation (Figure 1(b)) and echocardiography showed a large pericardial effusion measuring up to $2.6 \mathrm{cms}$ with the mitral inflow doppler consistent with tamponade physiology. Pericardial aspiration obtained $300 \mathrm{mls}$ of blood stained fluid which re-

"None of the authors have any conflicts of interest in relationship to this article. vealed gram positive cocci on staining and which grew staphylococcus aureus sensitive to Methicillin. Antimicrobial therapy was commenced in the form of Flucloxacillin 2 grams IV six hourly and Linezolid 600 mgs IV 12 hourly.

\section{CLINICAL COURSE}

Over the next 48 hours, the pericardium drained a further $500 \mathrm{mls}$ before the catheter was removed. A large left pleural effusion was subsequently drained. Computed tomography (CT) of the chest revealed both pleural and pericardial effusions with consolidation/collapse of the left lower lobe (Figure 1(c)). At this stage, the working diagnosis was of purulent pericarditis secondary to staphylococcal pneumonia. However, 6 days after admission she developed rapid atrial fibrillation and a new systolic murmur was noted. Repeat echocardiography revealed mitral regurgitation with an abnormality on the mitral valve.

Transesophageal echocardiography (TEE) was performed (iE33, Philips Healthcare, Reigate, Surrey, UK) and this demonstrated marked abnormality of the posterior mitral leaflet and the presence of a fistulous tract entering the left ventricular muscle below the posterior mitral leaflet and after a serpiginous course re-entering into the left atrium (Figures 2(a) and (b)). Further imaging was obtained with 64 slice CT (Aquilion 64, Toshiba Medical Systems, Tokyo, Japan). This demonstrated a pulsatile pseudoaneurysm of the left ventricle at the level of the mitral annulus with minimal tissue interposed between the apex of this pseudoaneurysm and the pericardial space (Figures 2(c) and (d)). Surgical opinions were obtained and the consensus opinion was to continue conservative management.

There were no current signs of infection of her left knee prosthesis. A bone scan revealed increased uptake involving the region of the knee replacement (Figure 


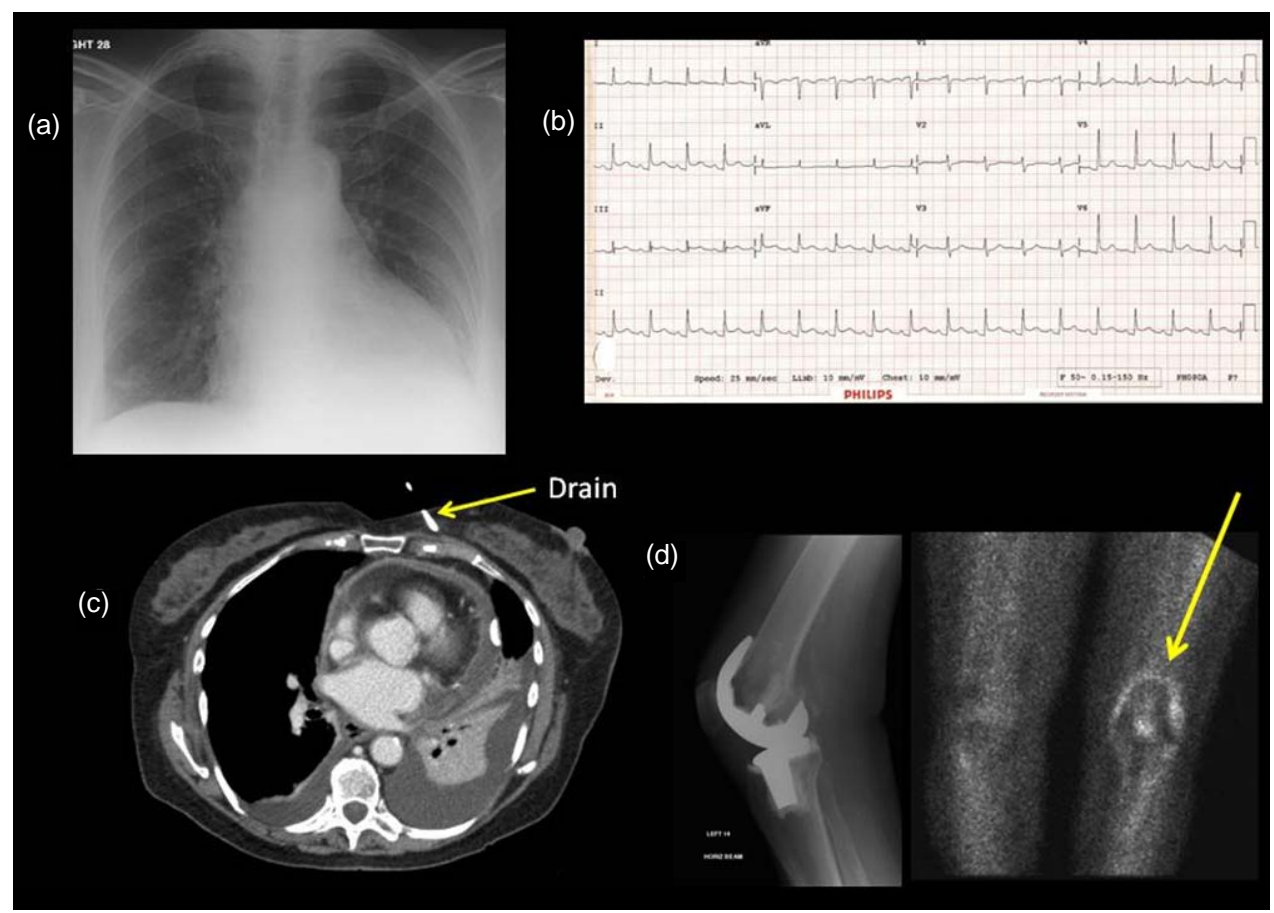

Figure 1. (a) Chest X Ray showing cardiomegaly; (b) EKG with diffuse ST segment elevation; (c) Computed tomography chest demonstrating left lower lobe consolidation, left pleural effusion, pericardial effusion with drain (yellow arrow) in situ; (d) X Ray and bone scan (Tc99) demonstrating left total knee prosthesis and increased uptake of technetium around prosthesis ( yellow arrow).

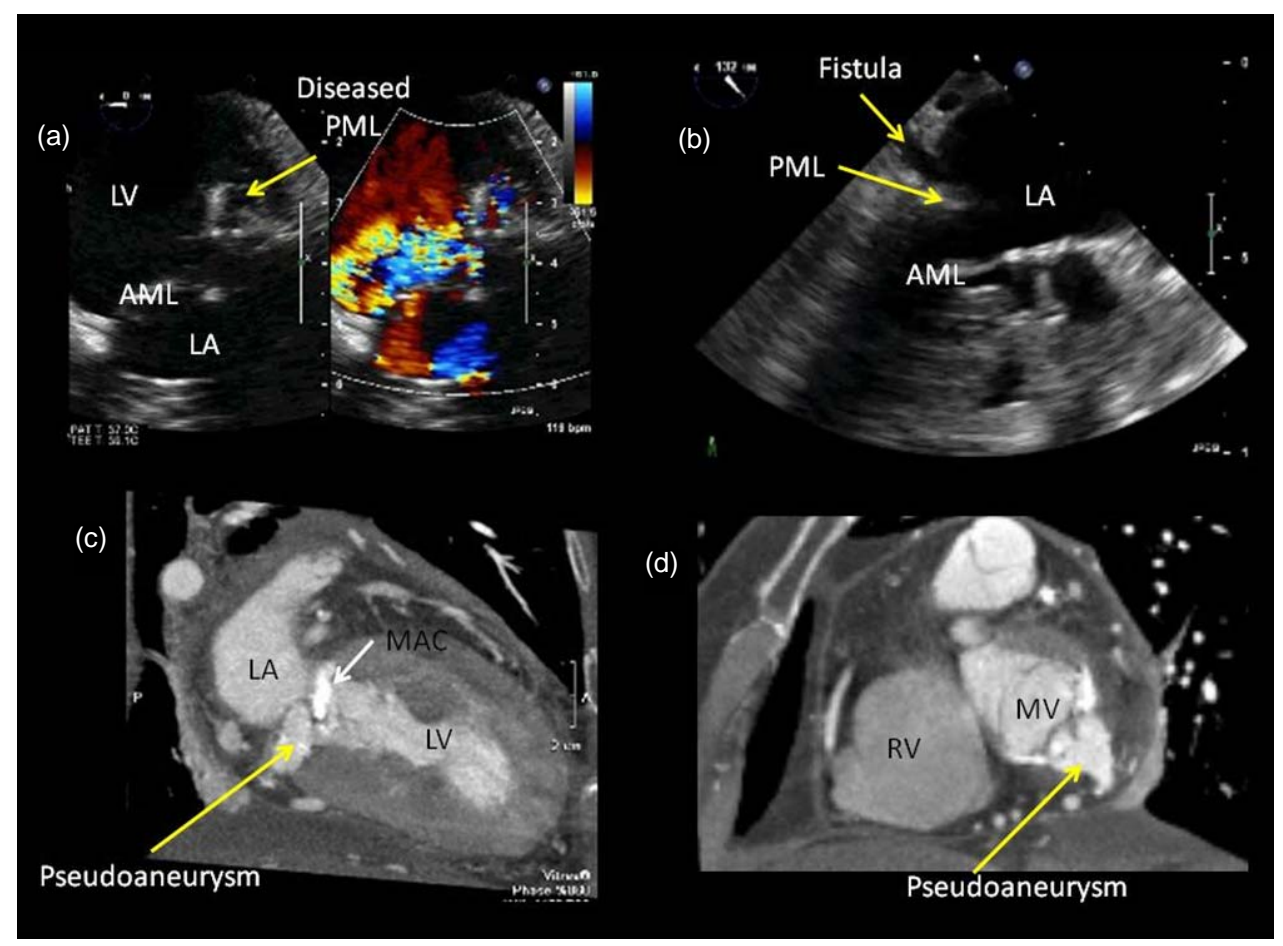

Figure 2. (a) TEE mid-esophageal 0 degrees image with and without color flow doppler demonstrating thickened and abnormal posterior mitral leaflet and significant eccentric mitral regurgitation; (b) Off axis TEE image highlighting entry of fistula into left atrium; (c) Cardiac CT image in long axis orientation showing LV pseudoaneurysm (yellow arrow) and mitral annular calcification (white arrow); (d) Cardiac CT short axis orientation demonstrating LV fistula and its relationship to mitral leaflets. 
1(d)). Orthopedic opinion was that the knee prosthesis was not infected and that bone scans can show non-specific increased uptake for at least 1 year following surgery.

The patient received 56 days of Flucloxacillin and 21 days of Rifampicin, intravenously and was discharged on oral therapy for a further 28 days. At follow up, there has been no clinical recurrence of infection but there is increasing left ventricular volume overloading secondary to severe mitral regurgitation.

\section{DISCUSSION}

A likely sequence of events in this patient is the development of bacteremia secondary to recurrent wound infection with seeding of the mitral valve. Repeated and prolonged oral antibiotics, prescribed for cellulitis of the left knee replacement, presumably partially treated endocarditis of the mitral valve but smouldering infection allowed the development of a fistula around the mitral annulus with seeding of the pericardium. Re-entry of the fistulous tract into the left atrium prevented catastrophic tamponade and led to the development of a localised pulsatile pseudoaneurysm. Multimodality imaging with TEE [2] and cardiac computed tomography [3] can help define complications of endocarditis and vigilant imaging should always be carried out in complex cases especially associated with staphylococcus aureus infection.

\section{REFERENCES}

[1] Katz, L.H., Pitlik, S., Porat, E., Biderman, P. and Bishara, J. (2008) Pericarditis as a presenting sign of infective endocarditis: Two case reports and review of the literature. Scandinavian Journal of Infectious Diseases, 40, 785791. doi:10.1080/00365540802169106

[2] Kunavarapu, C., Olkovsky, Y., Lafferty, J.C., Homayuni, A.R., Mohan, S.S. and McGinn, J. (2008) Unusual Complications of Staphylococcus aureus endocarditis. Journal of the American Society of Echocardiography, 21, 187e3$187 \mathrm{e} 5$.

[3] Prakash, S., Garg, N., Xie, G.Y. and Dellsperger, K.C. (2010) Giant left ventricular pseudoaneurysm. Journal of Cardiovascular Computed Tomography, 4, 284-285. doi:10.1016/j.jcct.2010.05.010 